\title{
Mobile Devices: Are They Tools or Toys for Saudi College Students?
}

\author{
Mahgoub Dafalla Ahmed \\ Department of English \& Translation \\ Faculty of Science \& Arts, Khulais Branch, University of Jeddah \\ P.O. Box: 80327, Jeddah 21589, Saudi Arabia \\ Tel: 966-534-053-521Ｅ-mail: mahgoub7700@ hotmail.com
}

Received: July 13, 2017

doi:10.5296/gjes.v3i2.11543
Accepted: August 18, $2017 \quad$ Published: August 25, 2017

URL: https://doi.org/10.5296/gjes.v3i2.11543

\begin{abstract}
The study aims at investigating Saudi college students' perceptions and attitudes towards the effectiveness of using mobile devices for academic purposes and other related activities. It tries to determine digital devices mostly used by students and measures to what extent they exploit these devices for academic purposes and in their other frequent activities. A survey was given to thirty-four Saudi university EFL students studying at the department of English and Translation, University of Jeddah, Saudi Arabia. The overall results have shown that most students always hold their mobile phones and bring them inside the classroom. Additionally, most of students have showed preference for using mobile devices for academic purposes and other related activities. The benefits of this study could be utilized for encouraging students to benefit from their mobile devices academically and designing future learning activities as well as mobile phone usage in the EFL classroom.
\end{abstract}

Keywords: Attitude, Digital devices, Mobile devices, Mobile phones, M-learning, Smartphones 


\section{Introduction}

Nowadays, all over the world, almost every teen owns or plans to have a digital device. It is noticeable everywhere, students are surfing the web, texting, watching or listening using some kind of digital devices. As a language practitioner, this question is always raised in my mind, do students benefit from these devices in their language learning or they use them just as toys?

The recent development in mobile technology, particularly the expansion of wireless network gives language learners greater chances to practice the language anytime and anywhere. Throughout the world, language teaching and learning has tended towards mobilization and personalization. Therefore, the main goal of this study is to explore the Saudi college learners' perceptions and attitudes towards using mobile phones in learning EFL, investigate how these devices are related with language learning and measure their advantages and drawbacks.

\section{Review of Literature}

\subsection{Theoretical Background}

Using mobile in teaching and learning languages is covered by many studies. Most of the studied (Rosell-Aguilar, 2007; Fallahkhair et al., 2007; Petersen \& Markiewicz, 2008; Liu et al., 2008; Cheng et al., 2010; Abdous et al., 2012; Oberg \& Daniels, 2011; Hsu, 2012), which have examined learners' attitudes and perceptions, agreed on the positive attitudes among learners towards mobile technology. Beres's (2011) study indicates to the positive response of students towards mobile learning encouraging learning outside classrooms. Nah, White and Sussex (2008) emphasize on student-centred learning and remind us about the enjoyment of students when they practise listening anywhere and anytime. Burston (2011) confirms the absolute positive attitudes of students towards mobile learning.

\subsubsection{Benefits of M-Learning}

Many researchers (Collins, 2005; Ogata et al., 2006; Kukulska-Hulme, 2006; Sarica \& Cavus, 2009; Guerrero et al., 2010) agree on the countless benefits EFL learners gain from using mobile phones. Mobile phones are multifunctional devices. The mobile phone can be used for writing, listening, watching videos, taking and sharing photos, surfing the web, downloading, uploading, etc. Klofer et al. (2002) lists five properties of mobile devices: 1) portability; 2) social interactivity; 2) context sensitivity; 4) connectivity; and 5) individuality. These properties have unlimited benefits. For example, portability and easy access to Web-sites facilitate exposition to authentic materials anytime and anywhere. In addition, mobile phones help students' interactions, develop social relationships and remove barriers (Lan et al., 2007; Lan, Sung, \& Chang, 2009) (Comas-Quinn et al., 2009); provide authenticity, and reduce anxiety (Horwitz, Horwitz, \& Cope, 1986). Mobile devices have an expanding social inclusion in language learning. Students can plan timed communications to share information and progress, and craft messages to resonate with different audiences - board of education members; teachers and staff; parents and peers. Moreover, mobile phones motivate students, encourage learner-centred and responsibility among learners (Csikszentmihalyi, 1992). They 
also help students to get feedback, be aware contextually, become independent learners (Attewell \& Webster, 2004; Chinnery, 2006; Klopfer, Squire, \& Jenkins, 2002; Soloway, Norris, Blumenfeld, Fishman, Krajcik, \& Marx, 2001) and enhance autonomy (Benson's (2007). Other benefits of these technologies are documentation of abroad experiences (Comas-Quinn et al., 2009) and taking and sharing photos (Wong, Chin, Tan, \& Liu, 2010).

\subsubsection{Challenges and Drawbacks of M-Learning}

In spite of the wide benefits, mobile devices have in language learning, significant challenges are preventing widespread effective implementation. Those challenges have centred largely on issues like reluctance of some administrations, teachers and students. Professional development should be no barrier at all to the use of good subject-oriented learning software. Administrators and teachers should remember that the students' minds are developing and changing with every passing fad. Other challenges represented in the lack of understanding or experience with mobile technology with some nontechnical students (Corbeil \& Valdes-Corbeil, 2007; Franklin, 2011); and anxiety (Kneebone et al., 2003). However, such problems can be easily reduced or eliminated as soon as students acquire more experience with mobile technology. Some researchers (Waycott 2004), (El-Hussein \& Cronje, 2010; Kalinic et al., 2011; Riad \& El-Ghareeb, 2008; Suki \& Suki, 2011) note to usability problems linked to small screen size and limited presentation of graphics, or difficulty entering data into the device (Smødral \& Gregory, 2003). The virtual keyboarding and one-finger data entry, and limited power cause other difficulties. Some potential drawbacks include limited nonverbal communications, limited message lengths, a lack of cultural context, and potentially limited social interaction. Connection problems are also concern: web-based language learners might choose to limit their online connection times, or they may not have access at all. However, these days the problem of disconnection is nearly solved because telecommunication companies provide web services nearly in hand for all users. However, some challenges posed by mobile learning represented in the weight and quick deletion of batteries especially when downloading applications (Riad \& El-Ghareeb, 2008); limited storage capacities and sites blocking. One more problem is that using mobile devices in classroom can cause distractions and interruptions (Cheon et al., 2012; Fried, 2008; Suki \& Suki, 2011). All students cannot focus their attentions and teachers cannot control each student's device to see the same information and images at the same time. Another concern seems to be about the challenged role of the teacher as the most learning activities take place outside the classroom (Sølverg \& Rismark, 2012). Other problems are taking photos of tests and instantly passing them on to other students; texting answers of tests to other students; privacy issues with teachers having personal phone numbers of students and vice versa and network dependency, some psychological barriers as well as challenges associated with studying in public venues or when using public transportation. The lack of available content specifically designed for language learning creates one major problem in using mobile phones. On the other hand, there are some researchers, who have sharp views against technology such as smartphones, tablets and other phone devices in language learning. For example, Stone (2004); Harley, Winn, Pemberton, and Wilcox (2007) argue, the main applications are suitable for administrative rather than pedagogical purposes. Beatty (2003, p. 


\section{MInstitute Macrothink $^{\text {Int }}$}

Global Journal of Educational Studies

ISSN 2377-3936

2017, Vol. 3, No. 2

72) warns claiming teachers need to be concerned about investing time and money in unproven technology.

\section{Questions of the Study}

1) What are the digital devices mostly used by Saudi college students? How often do Saudi students take their mobile phones with them?

2) What are the frequent activities in language learning Saudi students do with their mobile phones?

3) What are the advantages in language learning as perceived by Saudi college students from using mobile devices?

4) What are the drawbacks in language learning as perceived by Saudi college students from using mobile devices?

\section{Methodology}

\subsection{Subjects}

To identify the subjects for the study, the data was mainly collected from one subject. It consisted of 34 male students from the department of English and Translation, faculty of Science and Arts, University of Jeddah, Jeddah, Saudi Arabia. They were Saudi EFL learners from different levels. The study designed based on a baseline descriptive survey methodology. Accordingly, the students' perceptions and attitudes about using mobile phones as learning tools in formal education was described. Both qualitative and quantitative data were needed to answer the questions of the study. Students were asked for general information regarding name, university, faculty and level of study. They uncovered what digital devices they would like to use more and common uses of their mobile phones. Qualitative details about how participants used their mobile phones to support their learning will help broaden our understanding of the potential of mobile devices as aids to learning. Quantitative questions try to measure the level of enthusiasm felt by participants towards their mobile devices. The statements were designed to bring out the students' perceptions and attitudes towards using mobile phones in EFL learning. To identify what they think about learning using mobile phones, the students were asked to rank some statements, categorized into advantageous or disadvantageous according to their preferences.

\subsection{Measuring Instruments}

To obtain information about students' perceptions and attitudes towards using mobile devices in language learning, the researcher designed a questionnaire to be answered by students of English and Translation at the faculty of Sciences and Arts at University of Jeddah. The survey also sought information on students' frequent use of mobile devices to perform certain activities in learning EFL. The intention of designing this survey was to get abroad look at how Saudi students at University of Jeddah perceived the use of their devices as well as their attitudes for using it as a learning tool. To minimize any misunderstanding of the statements, he survey was offered in English and translated into Arabic orally by the investigator. It was 
divided into different parts. Firstly, participant students were asked to give their name and their level. Then, students were asked to determine which digital device they would like to use more in classes and outside classes, and how often they take their mobile phones with them. Secondly, the survey was designed according to the options of the three - point scale with "Always", "Sometimes", and "Never" in one part and "Agree", "Somewhat", and "Disagree" in the other part. There were 67 carefully prepared statements evaluating descriptive aspects of using mobile devices. Students provided answers on how mobile devices help and will help for college work, and in what tasks students prefer using these digital devices. Then, they were asked to rate their perceptions and attitudes towards using mobile devices for college work stating the advantages and the drawbacks.

Once the potential items were generated, reliability analysis was carried out to investigate the internal consistency of the items used in the survey. To verify and strengthen the validity, a pilot run of the instruments was found necessary for rewording ambiguous or poorly worded items and to eliminate unsuitable, or to add other appropriate items. The modification of the instruments in the light of the pilot run ensured that the instruments used in the main study were intelligible to the subjects. The survey was also examined by four faculty members who made valuable comments. No modifications worth noting were made. Only changes like substituting some vocabulary items were suggested to make sentences clearer. The Cronbach's Alpha reliability coefficient was calculated for the items, using the Likert-scale. The score was 0.90 .

\section{Data Analysis, Discussion and Results}

The focus of this part is to fulfil, discuss and state the results and findings. As mentioned before, the data was gathered from one instrument (a questionnaire). Therefore, the analysis, discussions and results of the data were treated by virtue of this instrument. In order to explore the role of the component variables, different statistical procedures were employed. Using the SPSS program, a descriptive statistics was used to measure the frequencies, mean and standard deviations of these variables. Statistical results in relation to hypotheses were drawn and discussed. Each hypothesis was restated and followed by an examination of the statistical results relating to it.

\subsection{Results Related to Question 1}

What are the digital devices mostly used by Saudi college students? How often do Saudi students take their mobile phones with them?

To answer these questions, a descriptive analysis is used to determine the frequency and the percentages. Results show the frequencies and percentages for students' levels, digital device mostly used by respondents for educational purposes and how often students hold their mobile phones. The usage varies widely by level. Table 1 and Graph 1 indicate that students at level 6 are the most students use digital devices in their learning (9 students, 26.5\%), followed by students at level 7 ( 8 students, $23.5 \%$ ), while levels 3 and 4 are the least students who use digital devices in their learning EFL (4 students, $11.8 \%$ for each). Results also show that the numbers skew slightly more toward smartphones. Smartphones are the most 


\section{Macrothink}

Global Journal of Educational Studies

ISSN 2377-3936

2017, Vol. 3, No. 2

frequently used devices among students (21 students, 61.8\%), and desktop computers are the least-used devices ( 1 student, 2.9\%) among the respondents to the survey. With regard to how often students take their mobile phones with them, (23 students, 67.6\%) say they always hold their mobile phones during classes, (7 students, 10\%) say they sometimes hold their mobile phones, and only (1 student, $2.9 \%$ ) says he never holds his mobile device with him.

Table 1. The Frequencies and percentages for the level, device used and held

\begin{tabular}{|l|l|l|l|l|l|l|l|l|}
\hline \multicolumn{3}{|c|}{ Level } & \multicolumn{3}{c|}{ Frequently Used Device } & \multicolumn{3}{l|}{ How often is the digital device held? } \\
\hline Level & Freq. & $\%$ & Device & Freq. & $\%$ & How often? & Freq. & $\%$ \\
\hline Level 3 & 4 & 11.8 & Desktop Computer & 1 & 2.9 & Always & 23 & 67.6 \\
Level 4 & 4 & 11.8 & Laptop Computer & 7 & 20.6 & Sometimes & 10 & 29.4 \\
Level 5 & 3 & 8.8 & Smartphone & 21 & 61.8 & Never & 1 & 2.9 \\
Level 6 & 9 & 26.5 & Tablet & 3 & 8.8 & & & \\
Level 7 & 8 & 23.5 & None & 2 & 5.9 & & & \\
Level 8 & 6 & 17.6 & & & & & 34 & 100 \\
\hline Total & 34 & 100 & & 34 & 100 & & & \\
\hline
\end{tabular}
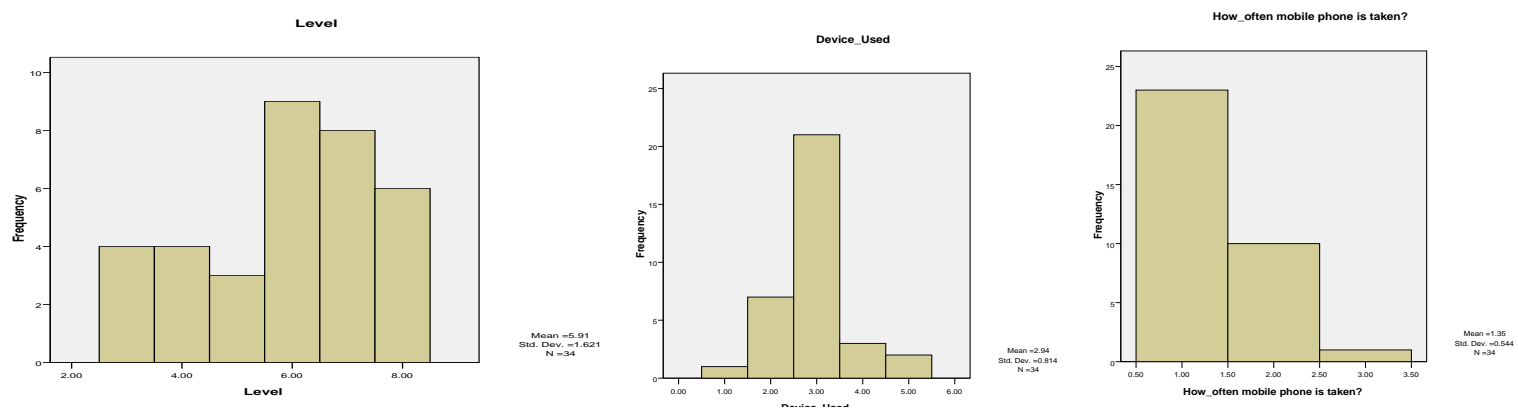

Figure 1. The Frequencies and percentages for the level of study, devices Used and Held

\subsection{Question 2}

What are the frequent activities in language learning Saudi students do with their mobile phones?

To answer this question, also a descriptive analysis is used to calculate the frequency, percentage, the means and standard deviations. Table 2 shows results for the frequent activities done by students using their mobile phones. 
Table 2. The Frequent activities students do with their mobile phones

\begin{tabular}{|c|c|c|c|c|c|c|c|c|}
\hline \multirow[b]{2}{*}{$\begin{array}{l}\text { Tasks preferred by students to be } \\
\text { done by mobile phones }\end{array}$} & \multicolumn{2}{|c|}{ Always } & \multicolumn{2}{|c|}{ Sometimes } & \multicolumn{2}{|c|}{ Never } & \multirow[t]{2}{*}{ Mean } & \multirow{2}{*}{$\begin{array}{c}\text { St. } \\
\text { Deviation }\end{array}$} \\
\hline & eFreq. & $\%$ & Freq. & $\%$ & Freq. & $\%$ & & \\
\hline 1. Homework & 13 & 28.2 & 20 & 58.8 & 1 & 2.9 & 1.65 & .54 \\
\hline 2. Research & 18 & 52.9 & 13 & 38.2 & 3 & 8.8 & 1.56 & .66 \\
\hline 3. Taking notes & 11 & 32.4 & 15 & 44.1 & 8 & 23.5 & 1.91 & .75 \\
\hline 4. Checking assignments & 10 & 29.4 & 22 & 64.7 & 2 & 5.9 & 1.76 & .55 \\
\hline 5. Reading digital English textbooks & 7 & 20.6 & 25 & 73.5 & 2 & 5.9 & 1.85 & .50 \\
\hline $\begin{array}{l}\text { 6. Reading English newspapers and } \\
\text { magazines }\end{array}$ & 8 & 23.5 & 19 & 55.9 & 7 & 20.6 & 1.97 & .67 \\
\hline 7. Checking class schedules & 13 & 28.2 & 17 & 50.0 & 4 & 11.8 & 1.74 & .67 \\
\hline 8. Taking exams & 6 & 17.6 & 17 & 50.0 & 11 & 32.4 & 2.15 & .70 \\
\hline 9. Listening to native speakers & 20 & 58.8 & 14 & 41.2 & 0 & 0.0 & 1.41 & .50 \\
\hline 10.Photos: storing, viewing or sharing & 19 & 55.9 & 12 & 35.3 & 3 & 8.8 & 1.53 & .66 \\
\hline 11. Recording videos and audios & 18 & 52.9 & 14 & 41.2 & 2 & 5.9 & 1.53 & .61 \\
\hline 12.Watching English YouTubes & 19 & 55.9 & 14 & 41.2 & 1 & 2.9 & 1.47 & .56 \\
\hline 13. Writing applications & 13 & 38.2 & 15 & 44.1 & 6 & 17.6 & 1.79 & .73 \\
\hline 14.Using a dictionary & 19 & 55.9 & 14 & 41.2 & 1 & 2.9 & 1.47 & .56 \\
\hline 15.Chatting in English & 13 & 38.2 & 19 & 55.9 & 2 & 5.9 & 1.68 & .59 \\
\hline 16. Writing e-mails & 15 & 44.1 & 17 & 50.0 & 2 & 5.9 & 1.61 & .60 \\
\hline $\begin{array}{l}\text { 17. Using social media: Facebook, } \\
\text { instagram, twitter, ... etc. }\end{array}$ & 24 & 70.6 & 9 & 26.5 & 1 & 2.9 & 1.32 & .53 \\
\hline 18.skyping & 9 & 26.5 & 11 & 32.4 & 14 & 41.2 & 2.15 & .82 \\
\hline 19.browsing the web & 19 & 55.9 & 13 & 38.2 & 2 & 5.9 & 1.50 & .62 \\
\hline 20.playing games & 16 & 47.1 & 16 & 47.1 & 2 & 5.9 & 1.59 & .61 \\
\hline 21.leisure and fun & 18 & 52.9 & 14 & 41.2 & 2 & 5.9 & 1.53 & .61 \\
\hline
\end{tabular}

As shown in Table 2, tasks always preferred by students to be done by their mobile phones include doing research, listening to native speakers, storing, viewing or sharing photos, recording videos and audios, watching English YouTubes, using dictionaries, using social media (Facebook, Instagram, Twitter, etc.), browsing the web, playing games and enjoying leisure and fun. However, tasks sometimes preferred by students to done by mobile phones include doing homework, taking notes, checking assignments, reading digital English textbooks, magazines and newspapers, checking class schedules, taking exams, writing applications, chatting and writing emails. However, the only task from the list students never prefer to do by their mobile phones is skyping.

\subsection{Results Related to Question 3}

What are the advantages in language learning as perceived by Saudi college students from using mobile devices? 
To answer this question, also a descriptive analysis is used to calculate the frequency, percentage, the means and standard deviations. Table 3 shows results for the frequency and percentages of benefits students gain from using mobile phones.

Table 3. The frequency and percentages of benefits students gain from using mobile phones

\begin{tabular}{|c|c|c|c|c|c|c|c|c|}
\hline \multirow[b]{2}{*}{$\begin{array}{l}\text { Benefits students gain from using } \\
\text { mobile phones in learning EFL }\end{array}$} & \multicolumn{2}{|c|}{ Always } & \multicolumn{2}{|c|}{ Sometimes } & \multicolumn{2}{|c|}{ Never } & \multirow[t]{2}{*}{ Mean } & \multirow{2}{*}{$\begin{array}{c}\text { St. } \\
\text { Deviation }\end{array}$} \\
\hline & Freq. & $\%$ & Freq. & $\%$ & Freq. & $\%$ & & \\
\hline 22.Effective tool for learning English & 25 & 73.5 & 8 & 23.5 & 1 & 2.9 & 1.29 & .52 \\
\hline $\begin{array}{l}\text { 23.Increase motivation for learning } \\
\text { English }\end{array}$ & 25 & 73.5 & 9 & 26.5 & 0 & 0 & 1.26 & .45 \\
\hline $\begin{array}{l}\text { 24. Change the way students learn in the } \\
\text { future }\end{array}$ & 23 & 67.6 & 9 & 26.5 & 2 & 5.9 & 1.38 & 60 \\
\hline $\begin{array}{l}\text { 25.Make learning interesting and } \\
\text { enjoyable }\end{array}$ & 24 & 70.6 & 9 & 26.5 & 1 & 2.9 & 1.32 & .53 \\
\hline $\begin{array}{l}\text { 26.Provide flexible learning anywhere, } \\
\text { anytime }\end{array}$ & 20 & 58.8 & 14 & 41.2 & 0 & 0 & 1.41 & .50 \\
\hline 27.Facilitate contextual learning & 13 & 38.2 & 20 & 58.8 & 1 & 2.9 & 1.65 & .54 \\
\hline $\begin{array}{l}\text { 28. Help students do better in } \\
\text { classrooms }\end{array}$ & 23 & 67.6 & 10 & 29.4 & 1 & 2.9 & 1.35 & .54 \\
\hline $\begin{array}{l}\text { 29. Help students do better outside } \\
\text { classrooms }\end{array}$ & 27 & 79.4 & 5 & 14.7 & 2 & 5.9 & 1.26 & .57 \\
\hline $\begin{array}{l}\text { 30. Expose students to English outside } \\
\text { classrooms }\end{array}$ & 19 & 55.9 & 15 & 44.1 & 0 & 0 & 1.44 & .50 \\
\hline $\begin{array}{l}\text { 31.Enhance student }- \text { student } \\
\text { interaction }\end{array}$ & 18 & 52.9 & 12 & 35.3 & 4 & 11.8 & 1.59 & .70 \\
\hline $\begin{array}{llll}\text { 32.Enhance } & \text { student } & - & \text { teacher } \\
\text { interaction } & & & \\
\end{array}$ & 17 & 50.0 & 16 & 47.1 & 1 & 2.9 & 1.53 & .56 \\
\hline $\begin{array}{l}\text { 33.Enhance student - administration } \\
\text { interaction }\end{array}$ & 20 & 58.8 & 12 & 35.3 & 2 & 5.9 & 1.47 & .61 \\
\hline 34.Make learning English easier & 20 & 58.8 & 13 & 38.2 & 1 & 2.9 & 1.44 & .56 \\
\hline $\begin{array}{l}\text { 35.Promote individualised learning } \\
\text { opportunities }\end{array}$ & 21 & 61.8 & 11 & 32.4 & 2 & 5.9 & 1.44 & .61 \\
\hline $\begin{array}{l}\text { 36.Promote lifelong learning } \\
\text { opportunities }\end{array}$ & 19 & 55.9 & 14 & 41.2 & 1 & 2.9 & 1.47 & .56 \\
\hline $\begin{array}{l}\text { 37.Encourage students to become } \\
\text { inquisitive learners }\end{array}$ & 20 & 58.8 & 13 & 38.2 & 1 & 2.9 & 1.44 & .56 \\
\hline $\begin{array}{l}\text { 38. Their portability offers comfort and } \\
\text { ease }\end{array}$ & 24 & 70.6 & 10 & 29.4 & 0 & 0 & 1.29 & 46 \\
\hline 39.Improve achievement & 19 & 55.9 & 13 & 38.2 & 2 & 5.9 & 1.50 & .62 \\
\hline
\end{tabular}




\begin{tabular}{|c|c|c|c|c|c|c|c|c|}
\hline $\begin{array}{r}\text { 40. Make students more active in } \\
\text { learning process }\end{array}$ & 23 & 67.6 & 10 & 29.4 & 1 & 2.9 & 1.35 & .54 \\
\hline $\begin{array}{l}\text { 41.Provide equal opportunities for } \\
\text { students to learn }\end{array}$ & 21 & 61.8 & 12 & 35.3 & 1 & 2.9 & 1.41 & .57 \\
\hline $\begin{array}{l}\text { 42. Help students have concentration on } \\
\text { lessons }\end{array}$ & 21 & 61.8 & 11 & 32.4 & 2 & 5.9 & 1.44 & .61 \\
\hline 43. Help students share information & 27 & 79.4 & 5 & 14.7 & 2 & 5.9 & 1.26 & .57 \\
\hline $\begin{array}{l}\text { 44.Improve engagement by offering a } \\
\text { more relax and comfortable setting } \\
\text { for learning }\end{array}$ & 20 & 58.8 & 13 & 38.2 & 1 & 2.9 & 1.44 & .56 \\
\hline $\begin{array}{l}\text { 45. Create enjoyable classroom and } \\
\text { remove the boredom of traditional } \\
\text { classroom practices }\end{array}$ & 19 & 55.9 & 13 & 28.2 & 2 & 5.9 & 1.50 & .62 \\
\hline 46.Can ensure feedback to all students & 14 & 41.2 & 19 & 55.9 & 1 & 2.9 & 1.62 & .55 \\
\hline 47. Reduce shyness & 19 & 55.9 & 11 & 32.4 & 4 & 11.8 & 1.59 & .70 \\
\hline $\begin{array}{l}\text { 48. Allow students to express } \\
\text { themselves in different ways }\end{array}$ & 21 & 61.8 & 10 & 29.4 & 3 & 8.8 & 1.47 & .66 \\
\hline 49.Provide privacy and freedom & 24 & 70.6 & 10 & 29.4 & 0 & 0 & 1.29 & .46 \\
\hline \begin{tabular}{|c|c|c|}
50. Provide immediate access to \\
learning materials
\end{tabular} & 22 & 64.7 & 11 & 32.4 & 1 & 2.9 & 1.38 & .55 \\
\hline $\begin{array}{l}\text { 51.Provide students with quicker } \\
\text { method for getting feedback }\end{array}$ & 19 & 55.9 & 11 & 32.4 & 4 & 11.8 & 1.59 & .70 \\
\hline \begin{tabular}{|c|c|c|}
$\begin{array}{c}\text { 52.Provide more robust and } \\
\text { personalised outcomes }\end{array}$ & \\
\end{tabular} & 18 & 52.9 & 14 & 41.2 & 2 & 5.9 & 1.53 & .61 \\
\hline $\begin{array}{l}\text { 53. Can be used for various special } \\
\text { features like podcasting }\end{array}$ & 20 & 58.8 & 11 & 32.4 & 3 & 8.8 & 1.50 & .66 \\
\hline 54. Can change the way students learn & 21 & 61.8 & 12 & 35.3 & 1 & 2.9 & 1.41 & .56 \\
\hline 55.Facilitate contextual learning & 19 & 55.9 & 14 & 41.2 & 1 & 2.9 & 1.47 & .56 \\
\hline $\begin{array}{l}\text { 56. Give students chances to follow their } \\
\text { progress }\end{array}$ & 23 & 67.6 & 9 & 26.5 & 2 & 5.9 & 1.38 & .60 \\
\hline
\end{tabular}

Note. Benefits from using mobile phones.

Results show respondents benefit a lot from using their mobile phones in most aspects of language learning. For most items of the list, the students declare they always benefit from mobile phones. In only two statements, students maintain that they sometimes benefit from their mobile phones. Statement number 22 which is about whether mobile phones are effective tool for learning English, 25 respondents (73.5\%) claim they always consider mobile phones are effective tools for learning English, 8 respondents (23.5\%) say mobile phones are sometimes effective tools and only 1 respondent (2.9\%) thinks he never considers mobile phones are effective tools for learning English. For statement number 23 which inquires if mobile phones increase motivation for learning English, 25 respondents $(73.5 \%)$ affirm mobile phones always increase motivation for learning English, 9 respondents $(26.5 \%)$ 
declare mobile phones sometimes do so, but there is no any respondent $(0 \%)$ thinks that mobile phones do not increase motivation for learning English. Statement number 24, which is about whether mobile phones change the way students learn in the future, 23 respondents (67.6\%) state mobile phones always change the way students learn, 9 respondents $(26.5 \%)$ maintain that mobile phones sometimes do that, and 2 respondents (5.9\%) argue mobile phones will not change the way they learn in the future. For statement number 25, which inquires about whether mobile phones make learning enjoyable and interesting, 24 respondents (70.6\%) agree mobile phones always make learning enjoyable and interesting, 9 respondents $(26.5 \%)$ think they sometimes do so, and only 1 respondent $(2.9 \%)$ thinks mobile phones do not make learning enjoyable and interesting. Statement number 26, which inquires whether mobile phones provide flexible learning anywhere and anytime, 20 respondents (58.8\%) maintain mobile phones always provide flexible learning anywhere and anytime, 14 respondents $(41.2 \%)$ say they sometimes do so, but there is no any respondent $(0 \%)$ thinks that mobile phones never provide flexible learning. About statement number 27, which asks if mobile phones facilitate contextual learning, 13 respondents $(38.2 \%)$ say that mobile phones always facilitate contextual learning, 20 respondents $(58.8 \%)$ maintain mobile phones sometimes facilitate contextual learning and only 1 respondent $(2.9 \%)$ states that mobile phones never facilitate contextual learning. Statement number 28, which inquires whether mobile phones help students do better inside classrooms, 23 respondents (67.6\%) assert mobile phones always help students do better inside classrooms, 10 respondents $(29.4 \%)$ declare that mobile phones sometimes help students do better inside classrooms and only 1 respondent $(2.9 \%)$ maintains that mobile phones never help students do better inside classrooms. For statement number 29, which is about if mobile phones help students do better outside classrooms, 27 respondents $(79.4 \%)$ state mobile phones always help students do better outside classrooms, 5 respondents $(14.7 \%)$ assert that mobile phones sometimes help students do better outside classrooms and 2 respondents think that mobile phones never help students do better outside classrooms. For statement number 30, which is about whether mobile phones expose students to English outside classrooms, 19 respondents (55.9\%) declare mobile phones always expose students to English outside classrooms, 15 respondents (44.1\%) maintain that mobile phones sometimes expose students to English outside classrooms, but there is no any respondent $(0 \%)$ says mobile phones never expose students to English outside classrooms. Statement number 31, which inquires whether mobile phones enhance student - student interaction, 18 respondents $(52.9 \%)$, state mobile phones always enhance student - student interaction, 12 respondents (35.3\%) maintain mobile phones sometimes enhance student - student interaction and 4 respondents (11.8\%) affirm mobile phones never enhance student - student interaction. Statement number 32, which inquires whether mobile phones enhance student - teacher interaction, 17 respondents $(50.0 \%)$, argue mobile phones always enhance student - teacher interaction, 16 respondents $(47.1 \%)$ declare mobile phones sometimes enhance student - teacher interaction and only 1 respondent $(2.9 \%)$ thinks mobile phones never enhance student - teacher interaction. Statement number 33, which inquires whether mobile phones enhance student - administration interaction, 20 respondents (58.8\%), assert mobile phones always enhance student - administration interaction, 12 respondents $(35.3 \%)$ maintain mobile phones sometimes enhance student - 
administration interaction and 2 respondents (5.9\%) affirm mobile phones never enhance student - administration interaction. Statement number 34, which asks if mobile phones make learning English easier, 20 respondents (58.8\%) think mobile phones always make learning English easier, 13 respondents (38.2\%) agree that mobile phones sometimes make learning English easier and only 1 respondent thinks that mobile phones never make learning English easier. Statement number 35, which is about whether mobile phones promote individualized learning opportunities, 21 respondents $(61.8 \%)$ declare mobile phones always promote individualized learning opportunities, 11 respondents $(32.4 \%)$ state that mobile phones sometimes promote individualized learning opportunities, and 2 respondents (5.9\%) maintain that mobile phones never promote individualized learning opportunities. Statement number 36, which inquires whether mobile phones promote lifelong learning opportunities, 19 respondents $(55.9 \%)$ assert that mobile phones always promote lifelong learning opportunities, 14 respondents $(41.2 \%)$ state mobile phones sometimes promote lifelong learning opportunities and only 1 respondent $(2.9 \%)$ thinks that mobile phones never promote lifelong learning opportunities. Statement number 37, which is about whether mobile phones encourage students to become inquisitive learners, 20 respondents $(58.8 \%)$ think that mobile phones always encourage students to become inquisitive learners, 13 respondents $(38.2 \%)$ agree that mobile phones sometimes encourage students to become inquisitive learners and only 1 respondent $(2.9 \%)$ asserts that mobile phones never encourage students to become inquisitive learners. Statement number 38, which asks if mobile phones' portability offers comfort and ease, 24 respondents $(70.6 \%)$ agree that mobile phones' portability always offers comfort and ease, 10 respondents $(29.4 \%)$ argue mobile phones' portability sometimes offers comfort and ease, but there no even a single respondent $(0 \%)$ thinks that mobile phones' portability never offers comfort and ease. Statement number 39, which is about whether mobile phones improve achievement, 19 respondents (55.9\%) say mobile phones always improve achievement, 13 respondents $(38.2 \%)$ state that mobile phones sometimes improve achievement, and 2 respondents (5.9\%) declare that mobile phones never improve achievement. Statement number 40, which inquires if mobile phones make students more active in learning process, 23 respondents $(67.6 \%)$ argue that mobile phones always make students more active in learning process, 10 respondents $(29.4 \%)$ assert that mobile phones sometimes make students more active in learning process and only 1 respondent $(2.9 \%)$ thinks mobile phones never make students more active in learning process. Statement number 41, which asks whether mobile phones provide equal opportunities for students to learn, 19 respondents $(55.9 \%)$ think mobile phones always provide equal opportunities for students to learn, 12 respondents $(35.3 \%)$ maintain that mobile phones sometimes provide equal opportunities for students to learn and only 1 respondent thinks that mobile phones never provide equal opportunities for students to learn. Statement number 42, which asks whether mobile phones help students have concentration on lessons, also 19 students (55.9\%) say mobile phones always have the power to do that, 11 respondents $(32.4 \%)$ state that mobile phones sometimes help students have concentration on lessons and 2 respondents $(5.9 \%)$ maintain mobile phones never help students have concentration on lessons. Statement number 43, which is about the role of mobile phones in sharing information, 27 respondents $(79.4 \%)$ think that mobile phones always help students share information, 5 respondents $(14.7 \%)$ 
agree that mobile phones sometimes help students share information and 2 respondents (5.9\%) argue mobile phones never help students share information. For statement number 44, which inquires if mobile phones improve engagement by offering a more relax and comfortable setting for learning, 20 respondents $(58.8 \%)$ declare that mobile phones always have the ability to improve engagement among students, 13 respondents $(38.2 \%)$ agree that mobile phones sometimes have the ability to improve engagement among students, and only 1 respondent $(2.9 \%)$ thinks that mobile phones never have the ability to improve engagement among students. Statement number 45, which asks whether mobile phones create enjoyable classroom and remove the boredom of traditional classroom practices, 19 respondents $(55.9 \%)$ confirm mobile phones always create enjoyable classroom and remove the boredom of traditional classroom practices, 13 respondents (38.2\%) argue that mobile phones sometimes create enjoyable classroom and remove the boredom of traditional classroom practices and 2 respondents $(5.9 \%)$ state that mobile phones never create enjoyable classroom and remove the boredom of traditional classroom practices. Statement number 46, which asks whether mobile phones can ensure feedback to all students, 14 respondents $(41.2 \%)$ declare that mobile phones can ensure feedback to all students, 19 respondents (55.9\%) assert mobile phones sometimes can ensure feedback to all students and only 1 respondent thinks that mobile phones cannot ensure feedback to all students. Statement number 47, which inquires whether mobile phones can reduce shyness, 19 respondents $(55.9 \%)$ agree mobile phones always can reduce shyness, 11 respondents (32.4\%) maintain that mobile phones sometimes reduce shyness, and 4 respondents $(11.8 \%)$ say that mobile phones never reduce shyness. Statement number 48, which inquires about whether mobile phones allow students to express themselves in different ways, 21 respondents (61.8\%) think mobile phones always allow students to express themselves in different ways, 10 respondents (29.4\%) assert mobile phones sometimes allow students to express themselves in different ways and 3 respondents $(8.8 \%)$ declare mobile phones never allow students to express themselves in different ways. Statement number 49, which asks if mobile phones provide privacy and freedom, 24 respondents $(70.6 \%)$ state mobile phones always provide privacy and freedom for learners, 10 respondents $(29.4 \%)$ agree mobile phones sometimes provide privacy and freedom for learners but there is no any respondent thinks that mobile phones never provide privacy and freedom. Statement number 50, which is about whether mobile phones provide immediate access to learning materials, 22 respondents (64.7\%) assert that mobile phones always provide immediate access to learning materials, 11 respondents $(32.4 \%)$ state that mobile phones sometimes provide immediate access to learning materials and there is only 1 respondent $(2.9 \%)$ thinks that mobile phones never provide immediate access to learning materials. For statement number 51, which inquires students if mobile phones provide students with quicker method for getting feedback, 19 respondents (55.9\%) declare that mobile phones always provide students with quicker method for getting feedback, 11 respondents $(32.4 \%)$ argue that mobile phones sometimes provide students with quicker method for getting feedback and 4 respondents (11.8\%) assert that mobile phones never provide students with quicker method for getting feedback. Statement number 52, which is about whether mobile phones provide more robust and personalised outcomes, 18 respondents $(52.9 \%)$ maintain that mobile phones always provide more robust and 
personalised outcomes, 14 respondents $(41.2 \%)$ declare that mobile phones sometimes provide more robust and personalised outcomes and 2 respondents (5.9\%) think that mobile phones never provide more robust and personalised outcomes. Statement number 53, which inquires about whether mobile phones can be used for various special features like podcasting, 20 respondents $(58.8 \%)$ say that mobile phones always can be used for various special features like podcasting, 11 respondents $(32.4 \%)$ agree that mobile phones sometimes can be used for various special features like podcasting and 3 respondents $(8.8 \%)$ declare that mobile phones cannot be used for various special features like podcasting. Statement number 54, which is about if mobile phones can change the way students learn, 21 respondents $(61.8 \%)$ think that mobile phones always can change the way students learn, 12 respondents $(35.3 \%)$ say that mobile phones sometimes can change the way students learn and only 1 respondent states that mobile phones cannot change the way students learn. Statement number 55, which is about whether mobile phones facilitate contextual learning, 19 respondents (55.9\%) agree that mobile phones always facilitate contextual learning, 14 respondents (41.2\%) argue that mobile phones sometimes facilitate contextual learning and only 1 respondent says that mobile phones never facilitate contextual learning. The last statement in this part, statement number 56, which asks if mobile phones give students chances to follow their progress, 23 respondents $(67.6 \%)$ agree that mobile phones always give students chances to follow their progress, 9 respondents $(26.5 \%)$ declare that mobile phones sometimes give students chances to follow their progress and 2 respondents (5.9\%) think that mobile phones never give students chances to follow their progress.

\subsection{Results Related to Question 4}

What are the drawbacks in language learning as perceived by Saudi college students from using mobile devices?

To answer this question, also a descriptive analysis is used to calculate the frequency, percentage, the means and standard deviations. Table 4 shows results for the frequency and percentages of drawbacks students think from using their mobile phones.

Table 4. The frequency and percentages of drawbacks students think from using mobile phones

\begin{tabular}{|c|c|c|c|c|c|c|c|c|}
\hline & \multicolumn{2}{|c|}{ Always } & \multicolumn{2}{|c|}{ Sometimes } & \multicolumn{2}{|c|}{ Never } & \multirow[t]{2}{*}{ Mean } & \multirow{2}{*}{\begin{tabular}{|c|} 
St. \\
Deviation
\end{tabular}} \\
\hline $\begin{array}{l}\text { Drawbacks students think from } \\
\text { using mobile phones in learning EFL }\end{array}$ & Freq. & $\%$ & Freq. $\%$ & $\%$ & Freq. & $\%$ & & \\
\hline $\begin{array}{l}\text { 57. Small, sensitive screen size is not } \\
\text { suitable for use in education }\end{array}$ & 19 & 55.9 & 12 & 35.3 & 3 & 8.8 & 1.53 & .66 \\
\hline 58.Distract students & 14 & 44.1 & 17 & 50.0 & 2 & 5.9 & 1.62 & .60 \\
\hline 59.Cause disciplinary problems & 18 & 52.9 & 12 & 35.3 & 4 & 11.8 & 1.59 & .70 \\
\hline $\begin{array}{l}\text { 60. The electromagnetic they emit } \\
\text { threaten human health }\end{array}$ & 14 & 41.2 & 16 & 47.1 & 3 & 8.8 & 1.94 & 1.72 \\
\hline $\begin{array}{l}\text { 61. Cause anxiety among students with } \\
\text { poor ICT literacy }\end{array}$ & 17 & 50.0 & 15 & 44.1 & 2 & 5.9 & 1.59 & .61 \\
\hline
\end{tabular}




\begin{tabular}{|l|l|l|l|l|l|l|l|l|}
\hline $\begin{array}{l}\text { 62.Limited memory make them } \\
\text { difficult to store data }\end{array}$ & 24 & 70.6 & 7 & 20.6 & 3 & 8.8 & 1.38 & .65 \\
\hline $\begin{array}{c}\text { 63.Limited battery life affect their use } \\
\text { in education }\end{array}$ & 22 & 64.7 & 11 & 32.4 & 1 & 2.9 & 1.38 & .55 \\
\hline 64.Security of data causes problems & 15 & 44.1 & 14 & 41.2 & 5 & 14.7 & 1.71 & .72 \\
\hline $\begin{array}{l}\text { 65.Using them in education requires } \\
\text { high costs }\end{array}$ & 15 & 44.1 & 14 & 41.2 & 5 & 14.7 & 1.71 & .72 \\
\hline $\begin{array}{l}\text { 66.Unavailability of m-learning } \\
\text { supported mobile phones }\end{array}$ & 17 & 50.0 & 12 & 35.3 & 5 & 14.7 & 1.65 & .73 \\
\hline 67.Poor networking incompatibility of mobile & 18 & 52.9 & 13 & 38.2 & 3 & 8.8 & 1.56 & .66 \\
\hline $\begin{array}{c}\text { 68. Language inces } \\
\text { phones }\end{array}$ & 20 & 58.8 & 11 & 32.4 & 3 & 8.8 & 1.50 & .66 \\
\hline
\end{tabular}

Note. Drawbacks from using mobile phones.

Table 4 shows positive results for the entire statements listed. Statement number 57, which is about the smallness of mobile phones and their sensitive screen sizes and their unsuitability for use in education, 19 respondents $(55.9 \%)$ claim they always consider mobile phones are small and their sensitive screen sizes are not suitable for use in education, 12 respondents (35.3\%) agree they sometimes consider mobile phones are small and their sensitive screen sizes are not suitable for use in education and 3 respondents $(8.8 \%)$ think they never consider mobile phones are small and their sensitive screen sizes are not suitable for use in education. For statement number 58 which inquires if mobile phones distract students, 14 respondents (44.1\%) agree mobile phones always distract students, 17 respondents (50.0\%) maintain mobile phones sometimes distract students and 2 respondents $(5.9 \%)$ say mobile phones never distract students. Statement number 59, which is about whether mobile phones cause disciplinary problems, 18 respondents (52.9\%) affirm mobile phones always cause disciplinary problems, 12 respondents $(35.3 \%)$ think mobile phones sometimes cause disciplinary problems and 4 respondents $(11.8 \%)$ say mobile phones never cause disciplinary problems. Statement number 60, which asks whether the electromagnetic mobile phones emit threaten human health, 14 respondents $(41.2 \%)$ argue that the electromagnetic mobile phones emit always threaten human health, 16 respondents $(47.1 \%)$ declare the electromagnetic mobile phones emit sometimes threaten human health and 3 respondents $(8.8 \%)$ think that the electromagnetic mobile phones emit never threaten human health. Statement number 61, which is about whether mobile phones cause anxiety among students with poor ICT literacy, 17 respondents $(50.0 \%)$ state mobile phones always cause anxiety among students with poor ICT literacy, 15 respondents $(44.1 \%)$ claim that mobile phones sometimes cause anxiety among students with poor ICT literacy and 2 respondents $(5.9 \%)$ think that mobile phones never cause anxiety among students with poor ICT literacy. Statement number 62, which inquires students whether limited memory in mobile phones make students difficult to store data, 24 respondents $(70.6 \%)$ maintain that limited memory in mobile phones always make it difficult for students to store data, 7 respondents (20.6\%) claim limited memory in mobile phones sometimes make it difficult for students to store data and 3 respondents $(8.8 \%)$ say 
that limited memory in mobile phones never make it difficult for students to store data. Statement number 63, which asks if limited battery life affects students' use of mobile phones in education, 22 respondents $(64.7 \%)$ state limited battery life of mobile phones always affects students' use of mobile phones in education, 11 respondents $(32.4 \%)$ affirm that limited battery life of mobile phones sometimes affects students' use of mobile phones in education, and there is only 1 respondent $(2.9 \%)$ thinks limited battery life of mobile phones never affects students' use of mobile phones in education. Statement number 64, which inquires about whether security of data causes problems, 15 respondents (44.1\%) agree security of data always causes problems, 14 respondents $(41.2 \%)$ assert security of data sometimes causes problems and 5 respondents (14.7\%) say security of data never causes problems. For statement number 65, which is about whether using mobile phones in education requires high costs, 15 respondents (44.1\%) agree using mobile phones in education always requires high costs, 14 respondents $(41.2 \%)$ claim that using mobile phones in education sometimes requires high costs and 5 respondents $(14.7 \%)$ maintain that using mobile phones in education never requires high costs. Statement number 66, which inquires about the unavailability of m-learning which support mobile phones, 17 respondents $(50.0 \%)$ affirm there is always unavailability of m-learning which support mobile phones, 12 respondents $(35.3 \%)$ assert there is sometimes unavailability of m-learning which support mobile phones and 5 respondents $(14.7 \%)$ think there is unavailability of m-learning which support mobile phones. Statement number 67, which inquires whether there is poor networking, 20 respondents (58.8\%) agree there is always poor networking, 11 respondents $(32.4 \%)$ maintain there is sometimes poor networking and 3 respondents $(8.8 \%)$ say there is no poor networking. About the last statement in this survey, statement number 68 , which asks about language incompatibility of mobile phones, 18 respondents $(52.9 \%)$ maintain there is always language incompatibility of mobile phones, 13 respondents $(38.2 \%)$ agree there is sometimes language incompatibility of mobile phones and 3 respondents $(8.8 \%)$ think there is no language incompatibility of mobile phones.

In accordance with most studies that investigate learners' perceived ease of use, perceived usefulness, intentions, and attitudes towards the use of mobile technologies for language learning, the results of this study have shown a positive attitude towards the use of mobile technologies for learning English as a foreign language.

\section{Conclusion}

The study showed that nearly all students bring mobile phones to the classroom and that there is tendency to use these devices to perform academic activities. They like the flexibility of this approach, capability to access learning materials and the interaction between themselves in one side and their teachers in the other side. However, a large number of students ignore this technology and its assistance to their learning. Therefore, certain polices have to be adopted in using mobile devices inside classrooms. For example, blending traditional teaching methods with new technology tools is creating student-centred classrooms that motivate students to succeed and increase their engagement in college. This innovative instructional method accommodates all students regardless of their learning style (Sharma and Barrett, 2007). 


\section{References}

Abdous, M., Facer, B. R., \& Yen, C. J. (2012). Academic effectiveness of podcasting: A comparative study of integrated versus supplemented use of podcasting in second language classes. Computers and Education, 58, 43-52. https://doi.org/10.1016/j.compedu.2011.08.021

Attewell, J., \& Webster, T. (2004). Engaging and supporting mobile learners. In J. Attewell, \& C. Savill-Smith (Eds.), Mobile learning anytime everywhere: A book of papers from MLEARN 2004 (pp. 15-20). London, UK: Learning and Skills Development Agency.

Beatty, K. (2003). Teaching and researching computer assisted language learning. Essex: Pearson Education.

Benson, P. (2007). Autonomy in language teaching and learning. Language Teaching, 40(1), 21-40. https://doi.org/10.1017/S0261444806003958

Beres, D. (2011). Mobile-assisted language learning from the student perspective: Encouraging effective language learning strategies outside of the classroom. In B. R. Facer, \& M. Abdous (Eds.), Academic podcasting and mobile assisted language learning: Applications and outcomes (pp. 93-110). Hershey, PA: IGI Global. https://doi.org/10.4018/978-1-60960-141-6.ch006

Burston, J. (2011). Exploiting the pedagogical potential of MALL. Retrieved from http://www.moblang.mobi/conference/files/PedagogicalAspectsOfMobileLearning_MobLang _JackBurston.pdf

Cheng, S. C., Hwang, W. Y., Wu, S. Y., Shadiev, R., \& Xie, C. H. (2010). A mobile device and online system with contextual familiarity and its effects on English learning on Campus. Educational Technology and Society, 13(3), 93-109.

Cheon, J., Lee, S., Crooks, S. M., \& Song, J. (2012). An investigation of mobile learning readiness in higher education based on the theory of planned behavior. Computers \& Education, 59, 1054-1064. https://doi.org/10.1016/j.compedu.2012.04.015

Chinnery, G. (2006). Going to the MALL: Mobile assisted language learning. Language Learning \& Technology, 10(1), 9-16. Retrieved from http://lit.msu.edu/vol10num1/emerging/default.html

Collins, T. (2005). English class on the air: Mobile Language Learning with Cell Phones. Proceedings of the Fifth IEEE International Conference on Advanced Learning Technologies (ICALT'05). https://doi.org/10.1109/ICALT.2005.137

Comas-Quinn, A., Mardomingo, R., \& Valentine, C. (2009). Mobile blogs in language learning: Making the most of informal and situated learning opportunities. ReCALL, 21, 96-112. https://doi.org/10.1017/S0958344009000032

Corbeil, J. R., \& Valdes-Corbeil, M. E. (2007). Are you ready for mobile learning? Educause Quarterly, 30(2), 51-58.

Csikszentmihayli, M. (1992). Flow: The Psychology of Happiness. London, U.K: Rider. 
El-Hussein, M. O. M., \& Cronje, J. C. (2010). Defining mobile learning in the higher education landscape. Educational Technology \& Society, 13, 12-21.

Fallahkhair, S., Pemberton, L., \& Griffiths, R. (2007). Development of a cross-platform ubiquitous language learning service via mobile phone and interactive television. Journal of $\begin{array}{llll}\text { Computer Assisted } & \text { Learning, 23(4), }\end{array}$ https://doi.org/10.1111/j.1365-2729.2007.00236.x

Franklin, T. (2011). Mobile learning: At the tipping point. The Turkish Online Journal of Educational Technology, 10(4), 261-275.

Fried, B. C. (2008). In-class laptop use and its effects on student learning. Computers \& Education, 50, 906-914. https://doi.org/10.1016/j.compedu.2006.09.006

Guerrero, L. A., Ochoa, S., \& Collazos, C. (2010). A mobile learning tool for improving grammar skills. Procedia Social and Behavioral Sciences, 2, 1735-1739. https://doi.org/10.1016/j.sbspro.2010.03.975

Harley, D., Winn, S., Pemberton, S., \& Wilcox, P. (2007). Using texting to support students' transition to university. Innovations in Education and Teaching International, 44, 229-241. https://doi.org/10.1080/14703290701486506

Horwitz, E. K., Horwitz, M. B., \& Joan, C. (1986). Foreign Language Classroom Anxiety. The Modern Language Journal (MLJ), 70(2), 125-132.

Hsu, L. (2012). English as a foreign language learners' perception of mobile assisted language learning: A cross-national study. Computer Assisted Language Learning, 1, 1-17.

Kalinic, Z., Arsovski, S., Stefanovic, M., Arsovski, Z., \& Rankovic, V. (2011). The development of a mobile learning applicafion as support for a blended e-learning environment. Technics Technologies Education Management, 6(4), 1345-1355.

Klopfer, E., Squire, K., \& Jenkins, H. (2002). Environment detectives: PDAs as a window into a virtual simulated world. In M. Milrad, U. Hoppe, \& Kinshuk (Eds.), Proceedings of IEEE International Workshop on Wireless and Mobile Technologies in Education (pp. 95-98). Växjö, Sweden: IEEE Computer Society. https://doi.org/10.1109/WMTE.2002.1039227

Kneebone, R., Nestel, D., Ratnasothy, J., Kidd, J., \& Darzi, A. (2003). The use of handheld computers in scenario-based procedural assessments. Medical Teacher, 25, 632-642. https://doi.org/10.1080/01421590310001605660

Kukulska-Hulme, A. (2006). Mobile language learning now and in the future. In P. Svensson (Ed.), Från vision till praktik: Språkutbildning och informationsteknik [From vision to practice: Language learning and information technology] (pp. 295-310). Sweden: Nätuniversitetet.

Lan, Y. J., Sung, Y. T., \& Chang, K. E. (2007). A mobile-devices-supported peer-assisted learning system for collaborative early EFL reading. Language Learning \& Technology, 11(3), 130-151. Retrieved from http://llt.msu.edu/vol11num3/pdf/lansungchang.pdf 


\section{Macrothink}

Global Journal of Educational Studies ISSN 2377-3936 2017, Vol. 3, No. 2

Lan, Y. J., Sung, Y. T., \& Chang, K. E. (2009). Let us read together: Development and evaluation of a computer assisted reciprocal early English reading system. Comwputers \& Education, 53(4), 1188-1198. https://doi.org/10.1016/j.compedu.2009.06.002

Liu, J., Yu, S., \& Ran, M. (2008). Research on the communicative mobile English learning model. Fifth IEEE International Conference on Wireless, Mobile, and Ubiquitous Technology in Education, 2008, 60-64. https://doi.org/10.1109/WMUTE.2008.26

McQuillan, J. (2006). iPod in education: The potential for language acquisition. Retrieved $\begin{array}{llll}\text { September } & 15, & 2012 & \text { from }\end{array}$ http://e2t2.binghamton.edu/pdfs/iPod_Lang_Acquisition_whitepaper.pdf

Oberg, A., \& Daniels, P. (2011). Analysis of the effect a student-centred mobile learning instructional method has on language acquisition. Computer Assisted Language Learning, 26(1), 177-196. http://dx.doi.org/10.1080/09588221.2011.649484

Ogata, H., Yin, C., Paredes, R. G., Saito, J. N. A., Yano, Y., Oishi, Y., \& Ueda, T. (2006). Supporting Mobile Language Learning outside Classrooms, proceedings of the Sixth International Conference on Advanced Learning Technologies (ICALT'06). https://doi.org/10.1109/ICALT.2006.1652595

Petersen, S. A., \& Markiewicz, J. K. (2008). PALLAS: Personalised Language Learning on Mobile Devices. Fifth IEEE International Conference on Wireless, Mobile, and Ubiquitous Technology in Education (pp. 52-59). https://doi.org/10.1109/wmute.2008.17

Riad, A. M., \& El-Ghareeb, H. A. (2008). A service oriented architecture to integrate mobile. Turkish Online Journal of Distance Education-TOJDE, 9(2), 200-219.

Rosell-Aguilar, A. (2007). Top of the pods: In search of a podcasting "pedagogy" for language learning. Computer Assisted Language Learning, 20(5), 471-492. https://doi.org/10.1080/09588220701746047

Sarica, G. N., \& Cavus, N. (2009). New trends in 21st Century English learning. Procedia Social and Behavioral Sciences, 1, 439-445. https://doi.org/10.1016/j.sbspro.2009.01.079

Sharma, P., \& Barrett, B. (2007). Blended Learning. Using Technology In and Beyond the Classroom. London: Macmillan.

Smørdal, O., \& Gregory, J. (2003). Personal digital assistants in medical education and practice. Journal of Computer Assisted Learning, 19, 320-329. https://doi.org/10.1046/j.0266-4909.2003.jca_033.x

Soloway, E., Norris, C., Blumenfeld, P., Fishman, B., Krajcik, J., \& Marx, R. (2001 June). Log on education: Handheld devices are ready-at-hand. Communications of the ACM, 44(6), 15-20. https://doi.org/10.1145/376134.376140

Sølvberg, A. M., \& Rismark, M. (2012). Learning spaces in mobile learning environments. Active Learning in Higher Education, 13(1), 23-33. https://doi.org/10.1177/1469787411429189 


\section{Macrothink}

Global Journal of Educational Studies

ISSN 2377-3936 2017, Vol. 3, No. 2

Stone, A. (2004). Mobile scaffolding: an experiment in using SMS text messaging to support first year university students. Proceedings of the IEEE International Conference on Advanced Learning Technologies (ICALT'04) (pp. 405-409). Joensuu, Finland. https://doi.org/10.1109/icalt.2004.1357446

Suki, N. M., \& Suki, N. M. (2011). Using mobile device for learning: From students' perspective. US-China Education Review, A1, 44-53.

Waycott, J. (2004). The Appropriation of PDAs as Learning and Workplace Tools, Unpublished PhD Thesis. The Open University, Milton Keynes, UK.

Wong, L. H., Chin, C. K., Tan, C. L., \& Liu, M. (2010). Students' personal and social meaning making in a Chinese idiom mobile learning environment. Educational Technology \& Society, 13, 15-26.

\section{Copyright Disclaimer}

Copyright for this article is retained by the author(s), with first publication rights granted to the journal.

This is an open-access article distributed under the terms and conditions of the Creative Commons Attribution license (http://creativecommons.org/licenses/by/3.0/). 